\title{
BMJ Open Defining and measuring health equity effects in research on task shifting interventions in high-income countries: a systematic review protocol
}

\author{
Aaron M Orkin, ${ }^{1,2,3}$ Allison McArthur, ${ }^{4}$ André McDonald, ${ }^{1}$ Emma J Mew, ${ }^{1}$ \\ Alexandra Martiniuk, ${ }^{1,5}$ Daniel Z Buchman, ${ }^{1,6}$ Fiona Kouyoumdjian,, ${ }^{7,8}$ \\ Beth Rachlis, ${ }^{1,9}$ Carol Strike, ${ }^{1}$ Ross Upshur ${ }^{1}$
}

To cite: Orkin AM, McArthur A McDonald A, et al. Defining and measuring health equity effects in research on task shifting interventions in highincome countries: a systematic review protocol. BMJ Open 2018;8:e021172. doi:10.1136/ bmjopen-2017-021172

- Prepublication history and additional material for this paper are available online. To view please visit the journal online (http://dx.doi.org/10.1136/ bmjopen-2017-021172)

Received 1 February 2018 Revised 25 May 2018 Accepted 20 June 2018
Check for updates

(C) Author(s) (or their employer(s)) 2018. Re-use permitted under CC BY-NC. No commercial re-use. See rights and permissions. Published by BMJ.

For numbered affiliations see end of article.

Correspondence to

Dr Aaron M Orkin;

aaron.orkin@mail.utoronto.ca

\section{ABSTRACT}

Introduction Task shifting interventions are intended to both deliver clinically effective treatments to reduce disease burden and address health inequities or population vulnerability. Little is known about how health equity and population vulnerability are defined and measured in research focused on task shifting. This systematic review will address the following questions: Among task shifting interventions in high-income settings that have been studied using randomised controlled trials or variants, how are health inequity or population vulnerability identified and defined? What methods and indicators are used to describe, characterise and measure the population's baseline status and the intervention's impacts on inequity and vulnerability?

Methods and analysis Studies were identified through database searches (MEDLINE, Embase, CINAHL, PsycINFO and Web of Science). Eligible studies will be randomised controlled trials published since 2004, conducted in highincome countries, concerning task shifting interventions to treat any disease, in any population that may face health disadvantage as defined by the PROGRESS-Plus framework (place of residence, race/ethnicity/culture/ language, occupation, gender/sex, religion, social capital, socioeconomic position, age, disability, sexual orientation, other vulnerable groups). We will conduct independent and duplicate title and abstract screening, then identify related papers from the same programme of research through further database and manual searching. From each programme of research, we will extract study details, and definitions and measures of health equity or population vulnerability based on the PROGRESS-Plus framework. Two investigators will assess the quality of reporting and measurement related to health equity and vulnerability using a scale developed for this study. A narrative synthesis will highlight similarities and differences between the gathered studies and offer critical analyses and implications. Ethics and dissemination This review does not involve primary data collection, does not constitute research on human subjects and is not subject to additional institutional ethics review or informed consent procedures. Dissemination will include open-access peer-reviewed publication and academic conference presentations. PROSPERO Registration Number CRD42017049959.
Strengths and limitations of this study

- First systematic review to examine how health inequity and population vulnerability are defined and measured in research on task shifting interventions.

- Advances a novel, sensitive search strategy for task shifting interventions evaluated using randomised controlled trial methods, while gathering supporting research from other methodologies.

- Draws on emerging frameworks for reviews on health equity and vulnerable populations.

- The review may face limitations related to quality assessment and study retrieval. Few studies may achieve high scores for study quality and rigour on health equity or population vulnerability measures.

\section{INTRODUCTION}

According to the WHO, task shifting involves the rational redistribution of healthcare tasks within health workforce teams. ${ }^{1}{ }^{2}$ Though it is not a new approach to health human resources shortages or healthcare delivery challenges, the term 'task shifting' was coined in the context of the HIV/AIDS crisis in Sub-Saharan Africa and has received substantially more attention from policy-makers, health authorities, clinicians and researchers in both low- and middle-income countries, and for delivering care to vulnerable or underserviced populations in more well-resourced settings. ${ }^{2-5}$

Task shifting addresses two parallel aims in the development and design of health systems. First, task shifting interventions address priority diseases with the intent to reduce morbidity and mortality. Systematic reviews now support a general consensus that task shifting can achieve this goal in underserved populations and deliver essential care for infectious diseases (eg, HIV/AIDS and tuberculosis), chronic and non-communicable 
diseases, maternal-child health and emergency care. ${ }^{3}$ 6-8 Task shifting from physicians to nurses has also been widely studied in high-income countries to increase access to a range of primary care interventions. ${ }^{9-11}$

Second, task shifting is intended to reduce health inequities, social gradients across populations or population vulnerabilities. Task shifting redistributes healthcare more equitably by decentralising healthcare services within communities, reducing reliance on the most highly trained and potentially least accessible professionals, and works around barriers that may separate people from conventional healthcare. ${ }^{1}$ The WHO and World Medical Association caution that task shifting may introduce a threat to health equity by improving access to care at the expense of quality, comprehensiveness and patient-centred care. $^{112}$ The World Medical Association asserts that task shifting should be viewed as an interim strategy to address inequity by delivering essential care to severely underserved populations, especially in settings where professional care is otherwise limited. ${ }^{12}$ Task shifting may redress inequities in access to care, but the intervention itself can have paradoxical effects on health equity by reducing or stratifying quality of care. In this context, it is reasonable to ensure that task shifting interventions achieve their intended goals to reduce health inequity by assessing effects on health equity when studying or evaluating task shifting interventions. Task shifting interventions should be justified and evaluated on both reductions in morbidity and mortality, and also their capacity to durably redress inequity or reduce population vulnerabilities.

There can be no certainty that task shifting interventions are effective at improving health equity unless the inequities of interest are identified and measured. WHO and the Cochrane Collaboration call for clarity and rigour in the definitions, measurement and reporting of health equity and equity-related outcomes. ${ }^{13-15}$ For most diseases addressed through task shifting interventions, measures of clinical effectiveness are well established. ${ }^{36-8}$ Consistent definitions and outcome measures with respect to population inequity and vulnerability are comparatively limited. ${ }^{16-18}$ The evaluation of health equity in research on task shifting could be enhanced with an accessible inventory of existing approaches to this problem.

This paper is a protocol for a systematic review to address the following questions: Among task shifting interventions that have been studied in high-income countries using randomised trials, how are health inequity or population vulnerability identified and defined? What methods and indicators are used to describe, characterise and measure the target population's baseline status and the intervention's impacts on the target population's inequity and vulnerability?

\section{METHODS}

Objectives

The primary objective of the review is to identify the working definitions of inequity and population vulnerability used in task shifting interventions in high-income countries evaluated with a randomised controlled trial or variant. The secondary objective is to identify how effects on inequity and vulnerability are measured and quantified in task shifting research in these settings. The review is therefore designed to describe and summarise the definitions and measures relevant to health inequity and population vulnerability among task shifting interventions in high-income countries. The review is not intended to summarise the effectiveness of task shifting to reduce health inequities or population vulnerability.

\section{Health equity and vulnerability}

We define health inequities as disparities in health or healthcare that are 'unnecessary, avoidable, unfair and unjust'. ${ }^{19}$ Inequity is distinct from inequality in that not all inequalities or disparities are unfair or unjustifiablefor example, males have prostate problems while females do not, but that inequality is not perceived as unfair. Equity is therefore rooted in principles of fairness and social justice, and the duty to reduce and redress those inequities takes on moral and ethical dimensions that are not intrinsic to all health disparities. ${ }^{20}$

We define vulnerability as an individual or population with an identifiably increased likelihood of incurring additional or greater wrong. ${ }^{21}$ Since vulnerabilities predispose an individual to wrongs or unjustifiable disparities, vulnerability is effectively a precondition for inequity, and correcting vulnerabilities may also reduce or redress inequities. Though some vulnerabilities are an intrinsic feature of an individual or population's status (such as children), other vulnerabilities are situational and present a greater likelihood of incurring wrong in one setting but not another (such as linguistic or cultural differences). The purpose and usage of identifying vulnerabilities in this context is not to label, disempower or stigmatise affected individuals or groups but to emphasise ethical obligations in healthcare and health services to protect from harm and respond to both the intrinsic and situational needs of those who are vulnerable. ${ }^{22}$

\section{Protocol registration and timeline}

We registered a standard version of this protocol through the International Prospective Register of Systematic Reviews (PROSPERO no. CRD42017049959) and will manage protocol amendments through this interface.

The Cochrane Handbook for Systematic Reviews of Interventions informs this protocol, as do the Preferred Reporting Items for Systematic Review and Meta-Analysis statements for protocols and equity-focused reviews (PRISMA-P and PRISMA-E). ${ }^{14}$ 23-25 These guidelines required some adaptation because this is a methodological and conceptual review rather than a review of an intervention or diagnostic tool.

Development of the complete protocol, search strategy review team and data management system occurred from January to December 2016. Reviewer training, title and abstract review, and full-text review occurred from 


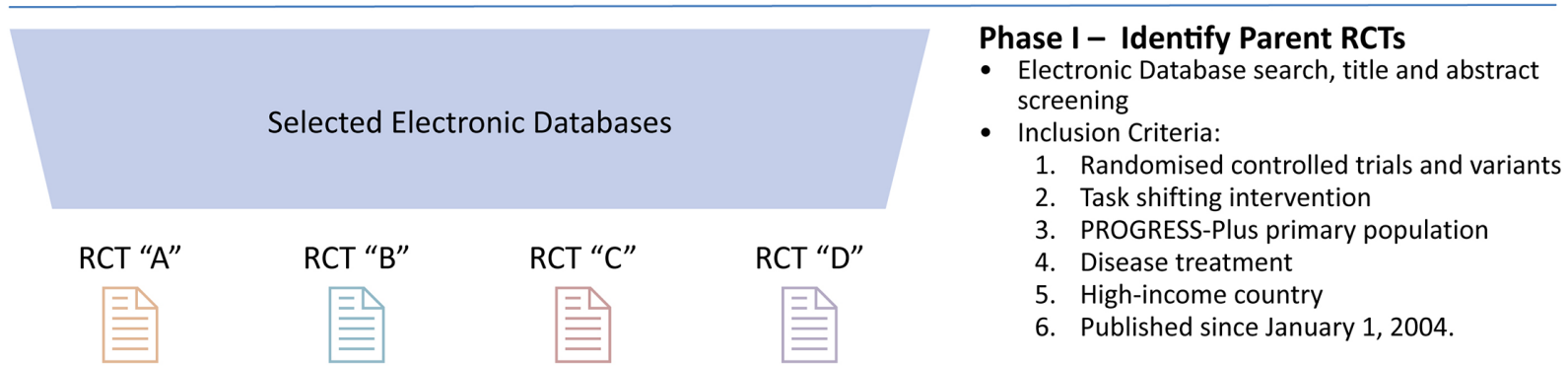

December 2016 to January 2018. Data extraction, analysis and manuscript preparation is anticipated to be complete by June 2018 .

\section{Patient and public involvement}

Patients and the public were not directly involved in the development of this protocol.

\section{Search strategy design}

All search strategies were developed in collaboration with a medical and public health information scientist (AMcA), which has been shown to improve systematic searching and review quality. ${ }^{26-28}$ Our search strategy is designed to retrieve all studies concerning task shifting interventions that have been evaluated at the level of a randomised controlled trial (RCT). To achieve this aim, our search and data extraction process will occur in three phases. Phase I will retrieve RCTs and RCT variants such as cluster-randomised trials or crossover trials, concerning task shifting interventions. Phase II will curate collections of related publications by identifying directly related non-RCT papers including process evaluations, embedded studies and secondary analyses published from the studies described in the parent papers. Phase III will involve the full-text review and data extraction of each publication collection (figure 1).

This approach retrieves all RCTs or RCT variants ('parent papers') concerning task shifting interventions, identifies related non-RCT papers concerning these programmes or projects ('child papers'), and bundles parent RCTs and child papers into publication collections ('families'). This novel approach restricts our review to task shifting interventions that have been evaluated with specific outcomes using methods that benefit from randomisation and a relatively high internal validity. This strategy also accounts for the fact that studies of a single complex intervention may involve multiple related publications. Investigators may consider inequity and vulnerability concepts in detail, but may not report those concepts in the RCT publication itself. For example, if an investigator group's long-term study on a single task shifting intervention has included a RCT, a qualitative research paper, an economic evaluation and a quality improvement study, we intend to first retrieve the parent RCT and then identify and include the other child publications all as a single 'publication family'.

\section{Phase I: randomised controlled trial retrieval}

We conducted electronic searches of the following biomedical and health sciences bibliographic databases for studies published between January 2004 and December 2016: MEDLINE, Embase, CINAHL, PsycINFO and Web of Science. We retrieved publications concerning in-progress trials using the Cochrane Central Register of Controlled Trials. All of the databases searched provided coverage for the date limits in question. 
The search strategy was developed according to the following procedure. We collected search terms from known studies and systematic reviews on task shifting as well as articles identified using PubMed's 'Related Articles' feature. ${ }^{6-8}$ We used these search terms to develop a preliminary MEDLINE search strategy and screened the results of this preliminary search to compile a validation set of relevant articles to test the precision and recall of terms and inform the selection of search terms.

We used a two-concept search strategy intended to identify papers containing at least one search term from each of the 'randomised trial' and 'task shifting' concepts. Natural language keywords were searched in the title, abstract and 'keyword heading' fields where available (see online supplementary appendix A for a list of terms). We refined and validated this search strategy to ensure that it retrieved 10 known studies concerning a subset of inclusion criteria from any study setting including both high-income and low-income and middle-income countries (see online supplementary appendix B).

We used an essentiality assessment process to evaluate recall and precision of all search terms within the task shifting concept. ${ }^{29}$ We considered a search term essential if it retrieved known relevant articles that could not be found using any other synonym through an iterative search process. The MEDLINE search strategy is included as online supplementary appendix A. We adapted the final MEDLINE search strategy to each database. Search adaptations favoured sensitivity over specificity where advanced search operators were unavailable.

\section{Study inclusion/exclusion}

Studies will be included in the review if they meet all of the following criteria (box 1):

1. RCTs and variants: this includes studies self-identified as RCTs or RCT variants such as cluster-randomised trials, crossover studies and factorial designs (online supplementary appendix A, lines 8-10). This excludes study protocols and abstracts retrieved without a complete manuscript.

2. Task shifting interventions: we will include studies describing 'task shifting' interventions, as defined by WHO: Task shifting involves the rational redistribution

\section{Box 1 Phase I 'parent RCT' inclusion criteria for} systematic review

1. Randomised controlled trial and variants.

2. Disease treatment.

3. Task shifting intervention.

4. PROGRESS-Plus ${ }^{*}$ primary population. ${ }^{27}$

5. High-income countries.

6. Health equity, vulnerability or identified synonym mentioned in the study title, abstract or discussion of the study population.

7. Published after 2004.

*PROGRESS-Plus: place of residence, race/ethnicity/culture/language, occupation, gender/sex, religion, social capital, socioeconomic position, age, disability, sexual orientation, other vulnerable groups of tasks among health workforce teams. Specific tasks are moved, where appropriate, from highly qualified health workers to health workers with shorter training and fewer qualifications in order to make more efficient use of the available human resources for health. ${ }^{1}$ Task shifting may involve moving or transforming healthcare interventions to workers with more limited training (such as community health workers) or more broadly to the lay public. The rational redistribution of tasks implies the transfer of existing health interventions to new workers and is distinguished from the rational addition of tasks, which involves adding new workers with new tasks to the health workforce team. For example, the introduction of case managers or care coordinators for patients with complex medical needs does not explicitly redistribute tasks but rather introduces a new worker or new layer of care to improve existing perceived deficiencies. Similarly, a trial comparing a usual-care control with usual care plus a community health worker intervention does not represent the redistribution of healthcare tasks unless the community health worker intervention displaces tasks generally undertaken in the usual care system.

Our search terms for the task shifting concept include a wide range of healthcare roles and task shifting terminology. We refer to 'task shifting' because it remains the dominant nomenclature for this concept in the academic and policy literature, while recognising that 'task sharing' and other emerging terminology may offer a more apt description of the interconnectedness and interdependence of diverse healthcare practitioners (online supplementary appendix A, lines 1-7).

3. PROGRESS-Plus primary population: we will include studies undertaken in any population that may face health disadvantage as defined by the PROGRESS-Plus framework (place of residence, race/ethnicity/culture/language, occupation, gender/sex, religion, social capital, socioeconomic position, age, disability, sexual orientation, other vulnerable groups). ${ }^{30}{ }^{31}$ For the purpose of this review, we do not consider the disease under study to define a vulnerability unless the study authors make that inequity explicit. For example, although mental health diagnoses may confer a relative disadvantage, a study concerning people with schizophrenia would not meet this inclusion criterion unless the health disadvantage of the population in question were made otherwise explicit. We further require that some aspect of the population's PROGRESS-Plus characteristics, health equity concerns, vulnerability or disadvantage be mentioned in the study title and abstract.

4. Disease treatment: we will include studies of interventions that provide treatment of already diagnosed or symptomatic disease. For example, studies concerning interventions such as cancer screening programmes in asymptomatic individuals, case-finding or public awareness campaigns without integrated disease treatment interventions will not be included. 
5. High-income countries: we will include studies undertaken in vulnerable populations from high-income countries according to the 2016 World Bank classification system. ${ }^{32}$ We place this restriction for two main reasons. First, by focusing on high-income settings, our review will be more specific to shifting tasks from existing health professionals to new or different cadres of health workers. In high-income settings, existing professionals might be deployed to address health needs, so engaging new cadres of health workers necessarily shifts tasks from within existing health workforce teams. In low-income or middle-income settings with more limited health professional workforces, task shifting often takes on the form of developing health systems and health workforce teams de novo, rather than shifting tasks away from existing professions. Second, inequity and vulnerability are relative concepts that have fundamentally different meanings and implications for task shifting research in low-income and middle-income countries versus high-income countries. In comparison with vulnerable subpopulations in well-resourced settings, the description of inequity and vulnerability in the literature concerning low-income and middle-income countries may be less explicit for the purposes of this review.

6. Date: we will include studies published since 1 January 2004. This date limitation avoids anachronistic assessments of studies published well before sentinel events in the scholarly history of task shifting and health equity, including the 2007 WHO Global Recommendations a Guidelines on Task Shifting, the 2008 PROGRESSPlus Framework, the 2008 Lancet Commission on the Social Determinants of Health and the 2010 Lancet Commission on Health Professionals for a New Century. ${ }^{114152833}$ A 2004 date limiter prevents the inclusion and unbalanced appraisal of studies undertaken prior to the development of contemporary definitions and conceptualisations of task shifting, vulnerable populations and health equity (online supplementary appendix A, line 13).

There will be no language restrictions. English search terms will be used to identify studies. Where English search terms yield study information in languages other than English, we will translate the title and abstract using Google Translate for title and abstract review, full-text review, data extraction and risk of bias assessment. ${ }^{34} 35$ There are no additional exclusion criteria.

\section{Additional search methods}

We will screen the references of relevant systematic reviews, the WHO recommendations and guidelines on task shifting, and the studies identified through the database searching. ${ }^{16-8}$ Other grey literature will not be included. We will link reports of the same study through examination of study details and will correspond with study authors to request further information as required.

We will update the MEDLINE search following the study selection and data extraction process. The lead investigators will review studies published between the original search and final manuscript submission using the same eligibility criteria.

\section{Information management, selection of studies}

De-duplicated bibliographic data will be uploaded to a custom-built website and data management system for screening papers. The website will be adapted from the systematic review interface developed by Orkin and colleagues using Google Sites. ${ }^{36}$ The interface will house the study protocol, reviewer training materials and other project information, and will allow customised access for each reviewer.

Reviewers will undergo training to ensure that the team is properly calibrated. Reviewers will be trained on a set of 100 citations, including 90 randomly selected citations from the database search results and 10 key studies meeting some or all inclusion criteria as identified by the lead investigator. Three reviewers will discuss and reach consensus on a gold standard set of answers. All reviewers will complete the training set, which will then be used to compute the sensitivity of the title and abstract review team. Review of the full set of papers will proceed if the review team has a greater than $95 \%$ sensitivity, assuming random assignments of papers and random pairings of reviewers. If this threshold is not reached, we will repeat the training exercise and further specify inclusion and exclusion criteria. Discrepancies will be addressed through review by the lead investigator, with access to the full-text manuscript where necessary. All parent RCTs retained after phase I will advance to phase II of the search.

\section{Phase II: curate publication families}

The goal of phase II is to curate publication families consisting of child papers that have been published from the same parent study. Each RCT included in phase I will be treated as a parent study and used to identify any child papers published from the same task shifting project. Using the same electronic bibliographic databases from phase I, we will retrieve non-RCT publications concerning only the task shifting projects studied in the RCTs that we included in phase I (see figure 1). For each RCT, we will extract author names and study identifiers such as the name of the programme under study. We will use these terms to search for publications of any kind that refer to the task shifting programmes or interventions that are studied in the parent RCTs. Search strategies will retrieve child papers, which are authored by the lead author and which contain at least one natural language keyword related to the title of the task shifting programme, study or intervention as described in the parent RCT. We will also use the 'related studies' and 'cited by' functions in bibliographic databases where available to curate these families. Phase II search strategies cannot be developed until phase I is complete because search term selection will be driven by bibliographic and descriptive terms of included parent RCTs, but we will report full phase II search strategies in the review. Phase II 
searches were restricted to studies published between 2004 and December 2017.

A publication will be considered to be a part of the same study collection as the parent RCT if it concerns the same task shifting programme, the same patient population or subpopulation, and the same setting. Each publication family will be curated under the leadership of an information scientist (AMcA).

\section{Phase III: full-text review and data extraction}

Two review authors will independently assess the full text of all studies included at phase II to ensure that:

1. Each publication collection includes a RCT that meets inclusion criteria (box 1).

2. All other papers are a part of a publication collection.

Disagreements at the full-text review stage will be resolved through a consensus discussion involving two reviewers and a lead investigator. We will report excluded studies in a 'Characteristics of Excluded Studies' table.

\section{Data extraction}

Two reviewers will independently extract data from each publication collection retained in the full-text review using a pre-piloted data extraction form, created using Google Sites. ${ }^{37}$ The following data will be extracted from each parent RCT:

1. Bibliographic information;

2. Study aim or question;

3. Study characteristics (design, sample size, number of arms);

4. Intervention and control (type and characteristics of interventions and control);

5. Study setting (country, region, community/level of health service), health condition and patient population (age, gender, ethnicity, language, other PROGRESS-Plus categories);

6. Outcome measures (type of outcome, definition of outcome, time of assessment); and

7. Results.

Outcome measures will be sorted into categories on the basis of their relevance to disease-specific outcomes or outcomes relevant to health equity and population vulnerability. For example, in a study on task shifting for the management of diabetes in a low-income population, outcomes concerning glycated haemoglobin levels or blood pressure would be identified as disease-specific. Measures of access to care or comparative assessments of conventional physician-led versus task shifted therapy would be identified as concerning health equity and vulnerability.

For each publication family, we will collect data on the types of studies included in the family and the study aims or questions. Across each publication family, we will extract any definition and identification of population, population vulnerability or health inequity being addressed through the task shifting intervention (especially PROGRESS-Plus population).

All data extractors will be familiar with the data collection form prior to extracting information from the
Table 1 Quality of equity and vulnerability definitions and outcome measurement

\begin{tabular}{|c|c|c|}
\hline Score & $\begin{array}{l}\text { Definition of health } \\
\text { equity or vulnerability }\end{array}$ & $\begin{array}{l}\text { Equity-relevant } \\
\text { outcome measure }\end{array}$ \\
\hline 1 & No definition & $\begin{array}{l}\text { No relevant outcome } \\
\text { measures }\end{array}$ \\
\hline 2 & $\begin{array}{l}\text { Study undertaken in a } \\
\text { population or setting } \\
\text { characterised through } \\
\text { the PROGRESS-Plus } \\
\text { framework } \\
\text { Inequity or vulnerability not } \\
\text { identified }\end{array}$ & $\begin{array}{l}\text { No relevant outcome } \\
\text { measures. Discussion } \\
\text { of equity implications of } \\
\text { other measures }\end{array}$ \\
\hline 3 & $\begin{array}{l}\text { Study undertaken in a } \\
\text { population or setting } \\
\text { characterised through } \\
\text { the PROGRESS-Plus } \\
\text { framework } \\
\text { Inequity and vulnerability } \\
\text { recognised but not clearly } \\
\text { defined or theorised }\end{array}$ & $\begin{array}{l}\text { Outcomes relevant to } \\
\text { alleviating or redressing } \\
\text { health effects of } \\
\text { PROGRESS-Plus } \\
\text { variables, but not } \\
\text { explicitly characterised } \\
\text { as relevant to equity }\end{array}$ \\
\hline 4 & $\begin{array}{l}\text { Targeted inequity and/or } \\
\text { population vulnerability } \\
\text { defined and explored }\end{array}$ & $\begin{array}{l}\text { Some outcomes } \\
\text { characterised as } \\
\text { relevant to alleviating or } \\
\text { redressing health effects } \\
\text { of PROGRESS-Plus } \\
\text { variables }\end{array}$ \\
\hline 5 & $\begin{array}{l}\text { Targeted inequity and/or } \\
\text { population vulnerability } \\
\text { defined and theorised } \\
\text { explicitly }\end{array}$ & $\begin{array}{l}\text { Study specifically } \\
\text { designed to measure } \\
\text { effects on health } \\
\text { inequity or reducing } \\
\text { vulnerability }\end{array}$ \\
\hline
\end{tabular}

included studies, and the form will be piloted using a random sample of studies. Where there are multiple reports on a single study, we will extract data from all reports into one data collection form with each citation listed. The lead investigator will check the information gathered for irregularities and will remedy discrepancies through discussion. We will record discrepancies and will synthesise recorded data from each investigator into a consensus data form.

\section{Quality: individual studies and across studies}

This review concerns the definition and measurement of health equity and vulnerability, rather than an assessment of the validity and reliability of study outcomes. Design and measurement factors leading to biases in the validity and reliability of the study outcomes are therefore less relevant to the review. In the context of this review, the analogue to conventional risk of bias assessments is the quality of defining and reporting outcomes with respect to health equity and population vulnerability.

Two reviewers will assign each study a score on a 5-point scale corresponding to the extent to which equity and vulnerability are defined and assessed in each task shifting intervention study (table 1). Disagreements will be resolved by consensus between two full-text reviewers. 
This scale will be validated through an internal validation study of 15 papers meeting inclusion criteria for this review. Reviewers conducting data extraction will assign a quality score to all 15 papers. We will then compute an inter-rater reliability for the quality scale, which will be included in the final manuscript. If the quality score is found to have poor inter-rater reliability, all scores will be assigned and justified on a consensus basis.

A summary table will compare the individual quality scores across studies. If the volume of included studies is adequate, we will compute and present summary and descriptive statistics regarding the quality of equity and vulnerability definitions and outcome measures across the included studies.

Measures of publication bias are not applicable to this review.

\section{Data synthesis and critical review}

We will summarise the included studies' characteristics and findings using a narrative approach because of the anticipated conceptual heterogeneity of studies. We will structure this narrative synthesis of results to highlight similarities and differences between studies. Specifically, we will group studies for comparison by the workers involved (community health workers, laypeople, nurses etc), the population of interest, the disease or condition of interest, and outcome measures. We will generate summary tables to underscore these groupings. An overall 'Summary of Findings Table' will present the definitions and usages of inequity and vulnerability in each trial.

\section{Conclusions}

Task shifting is a crucial strategy to deliver essential health services and redress health inequity among vulnerable and disadvantaged populations. ${ }^{12}$ Appropriate task shifting interventions must deliver both clinically effective interventions to reduce disease burden as well as reductions in health inequity or population vulnerability, but little is known about how these concepts are identified, defined and measured in task shifting research. The review arising from this protocol will deliver a first systematic description of the way in which health equity and vulnerability are defined and measured in task shifting research. The resulting inventory of definitions and measurements of health equity and population vulnerability may be used to improve the way in which these factors are measured and reported in studies on task shifting interventions.

\footnotetext{
Author affiliations

${ }^{1}$ Dalla Lana School of Public Health, University of Toronto, Toronto, Ontario, Canada ${ }^{2}$ Schwartz/Reisman Emergency Medicine Institute, Mount Sinai Hospital, Toronto, Ontario, Canada

${ }^{3}$ Department of Family and Community Medicine, University of Toronto, Toronto, Ontario, Canada

${ }^{4}$ Ontario Public Health Libraries Association, Toronto, Ontario, Canada

${ }^{5}$ George Institute for Global Health Australia, University of Sydney, Sydney, Australia ${ }^{6}$ University Health Network, Toronto, Ontario, Canada

${ }^{7}$ Centre for Urban Health Solutions, Li Ka Shing Knowledge Institute, St. Michael's Hospital, Toronto, Ontario, Canada
}

${ }^{8}$ Department of Family Medicine, McMaster University, Hamilton, Ontario, Canada ${ }^{9}$ Ontario HIV Treatment Network, Toronto, Ontario, Canada

Acknowledgements We thank Jeyasakthi Venugopal, Michelle Régnier and Sylvia Lyons for their support with manuscript formatting and submission.

Contributors AMO conceived of the study. AM0, AMCA, AMcD, EJM, AM, DZB, FK, $\mathrm{BR}, \mathrm{CS}$ and $\mathrm{RU}$ contributed to the conceptualisation and design of the proposed study and protocol. AMO, AMcA and AMCD wrote the first draft of the manuscript. All authors revised the presented manuscript, and reviewed and approved of the final submitted version of the manuscript.

Funding AMO is funded by the Canadian Institutes of Health Research Fellowship Program, the University of Toronto Department of Family and Community Medicine, and the Schwartz/Reisman Emergency Medicine Institute. AM is currently salary funded by a National Health and Medical Research Council (NHMRC) Translating Research into Practice (TRIP) Fellowship.

Competing interests None declared.

Patient consent Not required.

Provenance and peer review Not commissioned; externally peer reviewed.

Open access This is an open access article distributed in accordance with the Creative Commons Attribution Non Commercial (CC BY-NC 4.0) license, which permits others to distribute, remix, adapt, build upon this work non-commercially, and license their derivative works on different terms, provided the original work is properly cited, appropriate credit is given, any changes made indicated, and the use is non-commercial. See: http://creativecommons.org/licenses/by-nc/4.0/.

\section{REFERENCES}

1. World Health Organization. Task Shifting: Rational Redistribution of Tasks Among Health Workforce Teams: Global Recommendations and Guidelines. Geneva, Switzerland: WHO, 2008. (accessed 2016 May 25).

2. Chen L, Evans T, Anand S, et al. Human resources for health: overcoming the crisis. Lancet 2004;364:1984-90.

3. Callaghan M, Ford N, Schneider H. A systematic review of taskshifting for HIV treatment and care in Africa. Hum Resour Health 2010;8:8:8.

4. Heller R. Officiers de santé: the second-class doctors of nineteenthcentury France. Med Hist 1978;22:25-43.

5. Sidel VW. The barefoot doctors of the People's Republic of China. $N$ Engl J Med 1972;286:1292-300.

6. Kim K, Choi JS, Choi E, et al. Effects of community-based health worker interventions to improve chronic disease management and care among vulnerable populations: a systematic review. Am J Public Health 2016;106:671.

7. Lewin S, Munabi-Babigumira S, Glenton C, et al. Lay health workers in primary and community health care for maternal and child health and the management of infectious diseases. Cochrane Database Syst Rev 2010;3:CD004015.

8. Joshi R, Alim M, Kengne AP, et al. Task shifting for noncommunicable disease management in low and middle income countries-a systematic review. PLoS One 2014;9:e103754.

9. Maier CB, Aiken LH. Task shifting from physicians to nurses in primary care in 39 countries: a cross-country comparative study. Eur $J$ Public Health 2016;26:927-34.

10. Lovink MH, Persoon A, Koopmans R, et al. Effects of substituting nurse practitioners, physician assistants or nurses for physicians concerning healthcare for the ageing population: a systematic literature review. J Adv Nurs 2017;73:2084-102.

11. Laurant M, Reeves D, Hermens R, et al. Substitution of doctors by nurses in primary care. Cochrane Database Syst Rev 2005;2:CD001271.

12. World Medical Association. Resolution on task shifting from the medical profession. Ferney-Voltaire, France: World Medical Association, 2009. (accessed 16 May 2016).

13. PLOS Medicine Editors. Bringing clarity to the reporting of health equity. PLoS Med 2012;9:e1001334.

14. Welch V, Petticrew M, Petkovic J, et al. Extending the PRISMA statement to equity-focused systematic reviews (PRISMA-E 2012): explanation and elaboration. J Clin Epidemiol 2016;70:68-89.

15. Marmot M, Friel S, Bell R, et al. Closing the gap in a generation: health equity through action on the social determinants of health. Lancet 2008;372:1661-9. 
16. Dixon-Woods M, Cavers D, Agarwal S, et al. Conducting a critical interpretive synthesis of the literature on access to healthcare by vulnerable groups. BMC Med Res Methodol 2006;6:35.

17. Dixon Woods M, Kirk MD, Agarwal MS, et al. Vulnerable groups and access to health care: a critical interpretive review. Leicester, UK: National Coordinating Centre for NHS Service Delivery and Organisation Research and Development, 2005. (accessed 24 May 2016).

18. Flaskerud JH, Winslow BJ. Conceptualizing vulnerable populations health-related research. Nurs Res 1998;47:69-78.

19. Whitehead M. The concepts and principles of equity and health. Int J Health Serv 1992;22:429-45.

20. Braveman P, Gruskin S. Defining equity in health. J Epidemiol Community Health 2003;57:254-8.

21. Hurst SA. Vulnerability in research and health care; describing the elephant in the room? Bioethics 2008;22:191-202.

22. Clark B, Preto N. Exploring the concept of vulnerability in health care. CMAJ 2018;190:E308-E309.

23. Moher D, Shamseer L, Clarke M, et al. Preferred reporting items for systematic review and meta-analysis protocols (PRISMA-P) 2015 statement. Syst Rev 2015;4:1.

24. Welch V, Petticrew M, Tugwell P, et al. PRISMA-Equity 2012 extension: reporting guidelines for systematic reviews with a focus on health equity. PLoS Med 2012;9:e1001333.

25. Higgins JPT, Green S. Cochrane handbook for systematic reviews of interventions. Version 5.1.0. London. UK: The Cochrane Collaboration, 2011. (accessed 25 May 2016).

26. Alpi KM. Expert searching in public health. J Med Libr Assoc 2005;93:97-103.

27. Institute of Medicine of the National Academies. Standards for systematic reviews. Washington, DC: IOM, 2011. (accessed 25 May 2016).
28. Relevo $\mathrm{BH}$. Finding evidence for comparing medical interventions. Agency for Healthcare Research and Quality. Methods guide for effectiveness and comparative effectiveness reviews. Bethesda, MD: AHRQ, 2011.

29. Almanssori S. Creating a comprehensive search strategy for research on learning disabilities using the Pearl Harvesting Information Retrieval Framework. London, Ontario: University of Western Ontario, 2015:49.

30. O'Neill J, Tabish $\mathrm{H}$, Welch $\mathrm{V}$, et al. Applying an equity lens to interventions: using PROGRESS ensures consideration of socially stratifying factors to illuminate inequities in health. J Clin Epidemiol 2014;67:56-64

31. Kavanagh J, Oliver S, Lorenc T. Reflections on developing and using PROGRESS-Plus. Cochrane Health Equity Field and Campbell Equity Methods Group 2008;2:1.

32. World Bank. World Bank Country and Lending Groups. https:// datahelpdesk.worldbank.org/knowledgebase/articles/906519 (accessed Apr 2017).

33. Frenk J, Chen L, Bhutta ZA, et al. Health professionals for a new century: transforming education to strengthen health systems in an interdependent world. The Lancet 2010;376:1923-58.

34. Google. Google Translate. https://translate.google.ca/ (accessed Nov 2015).

35. Balk EM, Chung M, Chen ML, et al. Assessing the accuracy of Google Translate to allow data extraction from trials published in nonenglish languages. Rockville (MD: Agency for Healthcare Research and Quality (US), 2013.

36. Orkin AM, Curran JC, Fortune M, et al. Systematic review protocol: health effects of training laypeople to deliver emergency care in underserviced populations. BMJ Open 2016;6:e010609.

37. Google. Google Sites. https://apps.google.com/intx/en/products/ sites/ Accessed 2015 Nov. Supplementary Materials - ORKIN TSEquity.pdf 